\title{
Abnormal Frontostriatal Connectivity in Adolescent-Onset Schizophrenia and its Relationship to Cognitive Functioning.
}

Anthony James MB, BS, MRCP, MRCPsych, MPhil ${ }^{1,2^{*}}$, Eileen Joyce MA, PhD, FRCPsych MRCP $^{3}$, Daniel Lunn MA, DPhil, FRSS ${ }^{4}$, Morgan Hough BSc ${ }^{5}$, Louise Kenny BSc, D ClinPsych ${ }^{1}$, Pavandeep Ghataorhe BM, BCh, MA, MRCP ${ }^{6}$, Paul M Matthews MD, DPhil, FRCP ${ }^{7}$, Mojtaba Zarei MD, PhD, MRCP ${ }^{8}$,

1 Highfield Unit, Warneford Hospital, UK

2 Department of Psychiatry, Oxford University, Oxford, UK

3 Sobell Dept Motor Neuroscience, UCL Institute of Neurology, London, UK

4 Department of Statistics, University of Oxford, UK

5 FMRIB Centre, John Radcliffe Hospital Oxford, University of Oxford, UK.

6 GSK Clinical Imaging Centre, Hammersmith Hospital, London, UK

7 Division of Brain Sciences, Department of Medicine, Imperial College, London, UK

8 Institute of Cognitive Sciences Studies, Tehran, Iran

Corresponding Author:

A. James, Highfield Unit, Warneford Hospital, Oxford OX3 7JX.

email: anthony.james@psych.ox.ac.uk

Tel: +44 1865902682 Fax: +44 1865337413

Keywords: adolescence; schizophrenia: connectivity; striatum; frontal cortex; cognition. 


\begin{abstract}
Background. Adolescent-onset schizophrenia (AOS) is associated with cognitive impairment and poor clinical outcome. Cognitive dysfunction is hypothesised, in part, to reflect functional dysconnectivity between the frontal cortex and the striatum, although structural abnormalities consistent with this hypothesis have not yet been demonstrated in adolescence.
\end{abstract}

Objective To characterise fronto-striatal white matter white matter (WM) tracts in relation to cognition in AOS. Design. A MRI volumetric and diffusion tensor imaging study. Participants Thirty-seven AOS subjects and 24 age and sex matched healthy subjects Outcome measures Using probabilistic tractography, cortical regions with the highest connection probability for each striatal voxel were determined, and correlated with IQ and specific cognitive functions after co-varying for age and sex. Results. Bayesian Structural Equation modeling of FA (fractional anisotropy) from 12 fronto-striatal tracts showed processing speed to be an intermediary variable for cognition. AOS patients demonstrated generalised cognitive impairment with specific deficits in verbal learning and memory and in processing speed after correction for IQ. Dorsolateral prefrontal cortex connectivity with the striatum correlated positively with these measures and with IQ. DTI voxel-wise comparisons showed lower connectivity between striatum and the motor and lateral orbitofrontal cortices bilaterally, the left amygdalohippocampal complex, right anterior cingulate cortex, left medial orbitofrontal cortex and right dorsolateral prefrontal cortex.

Conclusions. Fronto-striatal dysconnectivity in large WM tracts that can explain core cognitive deficits are evident during adolescence. Processing speed, which is affected 

by alterations in WM connectivity, appears an intermediary variable in the cognitive deficits seen in schizophrenia. 


\section{Introduction}

Schizophrenia is a disorder of neurodevelopment(1) in which abnormalities give rise to psychotic symptoms and cognitive dysfunction. Functional neuroimaging studies strongly implicate the frontal cortex (2) yet few studies have found frontal cortical structural abnormalities in relation to cognition (3). MRI diffusion tensor imaging (DTI) tractography studies have found abnormal white matter tract integrity of the cingulum bundle (4), uncinate fasciculus (5), and superior longitudinal fasciculus (6) in relation to cognitive impairment. More specifically, in first episode schizophrenia (FES) white matter tract abnormalities (reduced FA and increased trace and radial diffusivity) between rostral medial frontal gyrus and rostral inferior frontal gyrus and the striatum have been found to be associated with executive dysfunction (7). There have been fewer studies of structural abnormalities and cognition in early-onset schizophrenia. However Epstein el al(8) found that lower FA in left inferior frontooccipital fasciculus and left inferior longitudinal fasciculus was associated with worse neurocognitive performance, a finding replicated in medication naive patients (9). A combined DTI and fMRI study of working memory in adolescent-onset schizophrenia revealed fMRI changes within the anterior cingulate and ventrolateral prefrontal cortex (hyperactivation) and the cuneus (hypoactivation), whereas DTI findings were of consistently reduced FA in the splenium and posterior cingulum.(10)

Altered functional connectivity and dopamine transmission in the striatum have been implicated in the cognitive impairment in schizophrenia (11). It was hypothesized that altered structural integrity of fronto-striatal white matter tracts (connectivity), contributes to the cognitive deficits seen in this disorder. In order to test this we applied DTI probabilistic diffusion tractography (12). Probabilistic tractography has 
advantages over conventional streamlining techniques in that it allows characterization of tracts even in areas of low fractional anisotropy, e.g. near their cortical or subcortical grey matter targets. Furthermore, probability density function (pdf) can be used as a robust, quantitative measure (strength) of structural connectivity between grey matter regions $(12,13)$.

Adolescent-onset schizophrenia is associated with more severe cognitive impairment and generally worse clinical outcomes than adult-onset schizophrenia $(14,15)$. It also offers the opportunity to test for clinico-pathological correlations without confounds from longer-term effects of medication and environmental factors of concern in adults. Here we are testing for fronto-striatal connectivities and their relationship to cognitive deficits in a well-characterised group of patients with AOS.

\section{Methods}

\subsection{Subjects}

Thirty-seven patients were recruited from adolescent units in the Oxford area. The Kiddie Schedule for Affective Disorders and Schizophrenia (16) was used to confirm a DSM V diagnosis of schizophrenia and the Positive and Negative Syndrome Scale (17) was used to assess symptom severity. All patients were receiving second generation antipsychotics (SGAs) (Table 1). Twenty-four healthy participants were recruited as controls through their general practitioners and were screened using the KSADS-PL for a history of emotional, behavioural or medical problems. All participants attended mainstream schools. 
Study exclusion criteria included moderate intellectual impairment (IQ <60), a history of pervasive developmental disorder, significant head injury, neurological disorder or major medical disorder. The Oxford Psychiatric Research Ethics Committee approved the study and written consent was obtained from all participants and parents.

\subsection{Neuropsychological assessments}

All participants were assessed for: 1) Full Scale IQ (FSIQ) using the Wechsler Abbreviated Scale of Intelligence (WASI) Vocabulary, Similarities, Block Design, Matrix Reasoning subtests (18) ; 2) verbal memory using the Word Lists subtests from the Children's Memory Scale in under 16s (19), and the Wechsler Memory Scale-III in over 16s (WMS/CMS) 1997) (20) ; 3) visuospatial skill and visuospatial memory with the Rey Complex Figure Test (RCFT) (21) ; 4) working memory with the Digit Span subtest from the Wechsler Intelligence Scale for Children-III UK (22) in under 16s and the Wechsler Adult Intelligence Scale-Revised (23); 5) executive function using Letter and Category Fluency subtests from the Delis-Kaplan Executive Function System (DKEFS) (24); and 6) processing speed using the Coding subtest from the Weschler Intelligence Scale for Children-III UK in under 16s (22) and the Digit Symbol task from the Wechsler Adult Intelligence Scale-Revised in over 16s (23). Handedness was assessed with the Edinburgh Handedness Questionnaire (25).

\subsection{Image acquisition}

All participants underwent whole-brain T1-weighted and diffusion-weighted scanning using a $1.5 \mathrm{~T}$ Sonata MRI scanner with a standard quadrature head coil and maximum

$40 \mathrm{mT} \mathrm{m}^{-1}$ gradient capability. Subjects were scanned with a 3D T1-weightedFLASH sequence using the following parameters: coronal orientation, matrix 256 x 256, 208 
slices, 1 x $1 \mathrm{~mm}^{2}$ in-plane resolution, slice thickness $1 \mathrm{~mm}$, TE/TR $=5.6 / 12 \mathrm{~ms}$, flip angle $\alpha=19^{\circ}$.

Diffusion-weighted images were obtained using echo-planar imaging (SE-EPI, TE/TR $=89 / 8500 \mathrm{~ms}, 60$ axial slices, bandwidth $=1860 \mathrm{~Hz} / \mathrm{vx}$, voxel size $2.5 \times 2.5 \times 2.5$ $\mathrm{mm}^{3}$ ) with 60 isotropically distributed orientations for the diffusion-sensitizing gradients at a $b$-value of $1000 \mathrm{~s} \mathrm{~mm}^{-2}$ and five $b=0$ images.

\subsection{Image Analysis}

Image analysis was carried out using tools from FSL (http://www.fmrib.ox.ac.uk/fsl) (26).

Seed and Target Masks: FIRST (part of FSL) was used to automatically segment subcortical grey matter structures (27) including the caudate, putamen and nucleus accumbens. These structures together form the corpus striatum that we used as the seed in tractography. Target masks were chosen according to their role in cognition (prefrontal cortex), motivation (medial temporal structures) and motor function (motor cortex). These structures included six regions: dorsolateral prefrontal cortex (DLPFC), medial orbitofrontal cortex (MOFC), lateral orbitofrontal cortex (LOFC), anterior cingulate cortex (ACC), and motor cortex (MC) as well as the amygdohippocampal complex (AHC). The hippocampus and amygdala were segmented using FIRST. MC included primary motor cortex, supplementary motor cortex and premotor cortex. For the creation of neocortical target masks, we used the Oxford-Harvard Cortical Atlas in standard space. Each mask was thresholded at 25\% and registered into native T1 image of each individual brain using FNIRT (non-linear 
registration tool - part of FSL). BET (part of FSL) was applied to remove non-brain tissue and FAST for tissue classification (CSF, white matter and gray matter) from the individual T1 brain image. All masks were then binarised. The individual cortical gray matter was multiplied by the neocortical masks, which had been registered into the individual brain space. This ensured that the masks were limited to the grey matter only.

Diffusion Tensor imaging and tractography: BET was used for brain extraction of the diffusion images. Affine registration matrix was calculated for transferring the masks into the diffusion space using FLIRT. Unconstrained probabilistic tractography was used to assess connectivity between seed and targets using the FMRIB Diffusion Tool (part of FSL). We calculated the probability of connectivity of each voxel in the seed with each voxel in the target masks separately. We then normalized the probability of connectivity in each voxel by changing the values to express the proportion of the total number of particles that reached any of the targets with connectivity value above 10 . The normalized images were then thresholded at 0.1 $(10 \%)$ to exclude spurious measures. This allowed us to create a connectivity-based segmentation of the seed mask as well as facilitated within subject and between-group comparison.

\subsection{Statistical Analyses}

Standardised scores were used for analysis of WASI FSIQ, Digit Span, Coding/Digit Symbol, DKEFS scores and for the WMS/CMS and RCFT memory scores. Analyses of demographic, clinical and neuropsychological measures were performed in STATA version 12.0 with t-test, ANCOVA and Chi-square as appropriate 
(28).Voxel-wise group comparison and regression analysis of connectivity strength was performed using Randomise (permutation analysis tool in FSL; number of permutations=5000). Threshold-Free Cluster Enhancement (TFCE) was applied to identify meaningful clusters without using an arbitrary level of thresholding (29). False discovery rate (FDR) was used for corrections for multiple comparisons with $\mathrm{p}<0.05(30)$.

In the regression model, normalised PDF (connectivity strength) was taken as the independent measure to predict neuropsychological variables. The frequency of voxel clusters and their voxel number were plotted. Clusters of less than 50 voxels were characteristic of artefactual registration mismatches at tissue contrast edges and were excluded. In all analyses IQ, age and gender were included as covariates except for the regression of connectivity strength predicting IQ itself when only age and gender were covariates.

Bayesian Structural Equation modeling (SEM)(31) was used to examine the putative strength of the relationship of fronto-striatal fibre connectivity and brain structures to processing speed - a cognitive function most characteristically found abnormal in schizophrenia (32). Two Bayesian structural models were constructed firstly with Coding and FSIQ combined as a bivariate response; and secondly with Coding as an intermediary variable to FSIQ . Inclusion in a regression model of FA measurements from 12 tracts would inevitably produce an unacceptable degree of over-parameterisation. Therefore, the dimension of the FA measurements was reduced by representing them as two orthogonal canonical FA factors, canV1 and canV2, which were the linear transformations of the raw FA measurements which maximised their correlation with the bivariate pair (Coding, FSIQ); canV1 correlation $=0.6729$, canV2 correlation $=0.5629($ Supplementary table 2$)$. 
Two Bayesian structural equation model (SEM) were constructed with can VI, canV2, white matter (WM), grey matter (GM) volumes, diagnosis, processing speed (Coding) and FSIQ, respectively (Figure 1,). The models were fitted using the Markov Chain Monte Carlo (MCMC) algorithm in the BUGS package (31). An initial burn-in of 30,000 was used, followed by 100,000 simulations; satisfactory convergence was achieved. Each model was fitted in two stages: the first stage including all variables; and the second being restricted to parsimonious models where coefficients with $95 \%$ credible intervals comfortably containing zero being set to zero.

\section{Results}

\subsection{Demographics and neuropsychology}

The two groups were matched for age, sex and handedness (Table 1). As expected, FSIQ was significantly higher in controls than AOS patients. In order to establish whether there were differences in specific cognitive functions independent of FSIQ, a group contrast was performed using FSIQ as a covariate. The variables surviving this analysis were: verbal learning $\left(\mathrm{F}_{(1,58)}=6.22, \mathrm{p}=0.016\right)$; verbal memory delayed recall $\left(\mathrm{F}_{(1,58)}=9.22, \mathrm{p}=0.004\right)$ and processing speed $\left(\mathrm{F}_{(1,58)}=4.29, \mathrm{p}=0.043\right)$. Subsequent examinations of the relationships between connectivity strength and cognitive function were confined to FSIQ and these specific neuropsychological measures.

\subsection{Neuroimaging}


Total white and cortical grey matter volumes were lower in AOS than in the healthy controls (Table 2). Analyses of individual cortical mask volumes revealed that this difference was driven by lower volumes for the AOS in motor and lateral orbitofrontal cortices bilaterally with left-lateralised differences in the dorsolateral prefrontal and anterior cingulate cortices. There were no volume differences between AOS and controls in any other structures measured.

\subsection{Bayesian Structural Equation Modeling}

The most parsimonious model revealed significant pathways with (i) Coding as an intermediary variable to FSIQ (ii) sex, white matter and canV2 acting directly upon Coding and only affecting FSIQ via Coding, (iii) diagnosis acting directly upon FSIQ and having no effect on Coding, (iv) grey matter having no significant effect on either Coding or FSIQ, (v) canV1 directly influencing both Coding and FSIQ (Figure 1). Table 3 here

Figure 1 here

\subsection{Cortico-striatal connectivity strength differences}

AOS patients showed lower connectivity than healthy controls between the striatum and the motor cortex and lateral orbitofrontal cortices, bilaterally, as well as between the striatum and in the left amygdalohippocampal complex and right anterior cingulate cortex (Figure 2). However, tracts between the striatum and the left medial orbitofrontal and right dorsolateral prefrontal cortices showed greater connectivity in the AOS patient group than in healthy controls (Figure 2).

\subsection{Brain structural correlates of neuropsychological impairment}


We tested the AOS group for parametric relationships between DTI measures of connectivity and those cognitive functional measures which distinguished them from controls (FSIQ, processing speed, verbal learning and verbal delayed recall memory).

Bilateral DLPFC-striatal connectivity showed a positive correlation with FSIQ within the AOS group (Figure 3). This relationship was not found in the control group. Verbal learning was positively correlated with connectivity between the striatum and the left DLPFC and right AHC and negatively correlated with its connectivity to the LOFC and MC bilaterally and the left MOFC (Figure 4). Verbal delayed recall memory was positively correlated with striatal connectivity to the left DLPFC and right $\mathrm{MC}$ and negatively with that to the right LOFC and MOFC and the left AHC (Figure 4). Processing speed showed positive correlations with striatal connectivity to the right DLPFC and left MC and negative correlations to the left LOFC and AHC and the right anterior cingulate (Figure 4).

\section{Discussion}

\subsection{Summary of findings}

Significant differences in the connectivity between the striatum and all frontal regions were found in AOS compared to healthy participants matched for age and sex. Abnormal connectivity in specific striatal regions corresponds to those known to receive projections from the frontal cortex in healthy subjects (33). AOS patients also demonstrated abnormal connectivity between dorsal striatum and the amygdalohippocampal complex and the anterior cingulate cortex. Connectivity was lower in patients for projections between the striatum and the amygdalohippocampal 
complex and the motor, lateral orbitofrontal and anterior cingulate cortices and greater for those between the striatum and the dorsolateral prefrontal and medial orbitofrontal cortices.

\subsection{Implication of Findings}

Our observations of a positive correlation of white matter connectivity strength between the striatum and the DLPFC with all of the cognitive measures studied are consistent with previous models of schizophrenia suggesting a pathophysiologically primary deficit in frontal-striatal outputs underlying the cognitive impairments in the disorder such as executive function, learning and memory $(34,35)$. Functional neuroimaging studies have strongly implicated dysfunctional DLPFC in the mediation of cognitive impairment in schizophrenia (36). Recent work in healthy volunteers suggests that DLPFC is a key node in a network active when executive or cognitive control of diverse psychological operations is required for the performance of demanding tasks (37) and is consistent with observations in the normal and lesioned brain that have shown IQ deficits in association with bilateral DLPFC dysfunction (38). While several studies using fractional anisotropy as an index of white matter structural integrity have found correlations with different measures of cognitive function in tracts linking frontal cortex and other cortical areas e.g., the cingulum bundle, uncinate fasciculus and superior longitudinal fasciculus (4-6), this study demonstrates a relationship between the structural connectivity of this major frontal outflow tract mediating executive control of behaviour and the typical profile of cognitive impairment seen in schizophrenia.

The full range of cognitive deficits characteristic of schizophrenia may be related to this pathology. Fry and Hale (39) provide evidence that during development, 
intelligence, working memory and information processing speed change together and that an age-related cognitive 'cascade' links these functions so that improved intelligence depends on improved working memory, which in turn is mediated by improved processing speed. Studies of the association between age-related fractional anisotropy, IQ and processing speed are consistent with this concept $(40,41)$. As processing speed is a basic cognitive operation fundamental to the performance of many higher order tasks, it is likely that the DLPFC-striatal white matter projections support the processing speed requirements for the execution of the tasks used in our study to assess IQ and verbal memory, as well as the processing speed task itself.

Interpretations of the connectivity findings here need to be in the context of neurodevelopmental processes. . The striatum in schizophrenia is an active region of development during adolescence, with a specific age-related increase in overall striatal volume (42), and localised shape changes along the ventral-medial- anterior aspect of the head the striatum (43). More generally, the connections of the striatum vary with the ventral portions of the striatum, which have predominantly limbic cortical connections (44), showing strong negative relationships with age, while dorsal portions, which have predominantly executive and motor cortical connections showing weaker positive relationships with age (42).

The finding of altered connectivity and FA and its relation to IQ appears, in part, to be mediated by processing speed. In the Bayesian structural equation model (SEM) (Figure 1), processing speed best fits as an intermediary variable between FA and IQ. Processing speed is thought to be a fundamental process in many executive functions, and possibly a core deficit in schizophrenia (45), so alterations in white matter 
connectivity, as measured by altered diffusion indices, make intuitive sense.

Furthermore, WM and sex were suggested to influence processing speed (Figure 1).

The finding that sex may influence processing speed, and hence IQ, has some support: female superiority in processing speed has been noted in the general population (46), and in those with schizophrenia (47), and has been shown to differentially underlie the lesser memory impairments seen in females with schizophrenia (48).

The finding of increased connectivity in certain tracts may seem surprising as previous studies of white matter in early-onset schizophrenia have reported reduced fractional anisotropy (FA) in white matter (49). However, FA is a relative measure of a local diffusion of water representing white matter integrity. Probabilistic tractography relates to FA but provides a more sensitive and specific measure because both white matter integrity and orientation are integrated over the entire pathway connecting two pre-defined regions of interest. A study using similar methodology to ours found both increased and decreased connectivity in different tracts linking thalamus and the cerebral cortex (50). A recent review (51) highlighted the increasing number of reports of increased FA in WM tracts in schizophrenia, while a study by Zhang et al(52) found a complex pattern of increased and decreased connectivities between cortical and subcortical structures, as demonstrated here. Nevertheless, the question of higher connectivity is difficult to distinguish as either lower confounds from crossing fibres or higher density of parallel fibres. Further technological development is required to help clarify this.

\subsection{Limitations}

Another limitation of this study is that the patients were medicated. Corticostriatal functional dysconnectivity in psychosis could be a state-dependent phenomenon, with 
increased functional connectivity of the striatum to the prefrontal and limbic regions being associated with improvement in symptoms following antipsychotic treatment (53). Furthermore, some of the patients were treated with clozapine, which has been shown to increase FA (54). However, we found no relationship between connectivity strength and medication dosage.

\subsection{Conclusions}

These findings in AOS provide evidence that abnormalities in connectivity between frontal cortex and striatum are a fundamental pathological characteristic of schizophrenia. Because fronto-striatal tracts are thought to mediate the processing of information that ultimately motivates and controls action selection, this finding is important for understanding the aetiology of schizophrenia cognitive impairments. Consistent with a growing literature, we found that the cognitive impairments were related to the connectivity strength of striatal projections from dorsolateral prefrontal cortex. As hypothetical mechanisms of cognitive impairment in schizophrenia suggest that DLPFC synaptic pathology causes aberrant glutamate and dopamine neurotransmission (34), our findings suggest that a better understanding of the abnormalities of DLPFC and its output circuitry are central to the development of treatments to improve cognitive function in schizophrenia. However, our study also highlighted the importance of pathology reflected in mesial temporal and orbitofrontal cortical connectivities for cognition, suggesting a functional-anatomic substrate for contributions of abnormal information salience and impaired sensorimotor inhibition to the expression of cognitive deficits in the disorder. 


\section{Figure captions}

Figure 1: Bayesian Structural Model. Examination of the relationship of processing speed (Coding) to full scale IQ (FSIQ) and the variables of wm - white matter volume, gm- grey matter volume, sex, diagnosis and the canonical variates - canVI, canV2 of the fractional anisotropy in the frontostriatal tracts.

Figure 2: Coronal, sagittal and axial views. Group comparison of striatal connectivity contrasting controls and adolescent onset schizophrenia. Red/yellow voxels are where connectivity in controls is greater than patients; blue voxels are where connectivity in patients is greater than controls. ACC - anterior cingulate cortex; DLPFC dorsolateral prefrontal cortex; Hpc - Amygdalo-hippocampal complex; LOFC lateral orbitofrontal cortex; MOFC - medial orbitofrontal cortex; Motor - motor cortex.

Figure 3: Coronal, sagittal and axial views of voxels where IQ positively correlates with DLPFC-striatal connectivity in schizophrenia.

Figure 4. Coronal view of voxels where digit-symbol coding (left column) verbal learning (middle column) and delayed verbal recall memory (right column) correlate with cortico-striatal connectivity in schizophrenia. Blue and yellow voxels represent negative and positive correlations respectively. ACC - anterior cingulate cortex; DLPFC - dorsolateral prefrontal cortex; Hpc - Amygdalo-hippocampal complex; LOFC - lateral orbitofrontal cortex; MOFC - medial orbitofrontal cortex; Motor motor cortex. 


\section{Disclosure of interest:}

Professor Matthews was an employee of GlaxoSmithKline and holds stocks in that company. Prof Zarei received consultation fee from GlaxoSmithKline on this project. All other authors- Professor Joyce, Drs James, Lunn, Kenny and Ghataorhe and Mr Hough report no competing interests.

\section{Acknowledgements}

We are grateful to the participants and their families and the Donnington Health Centre, Oxford. We would also like to thank Susan James for her work. PMM is an NIHR Senior Investigator and is grateful the Imperial College Healthcare Trust Biomedical Research Centre for support.

\section{Funding}

Support for this study was provided by the Medical Research Council, UK (G0500092) (AJ), the Oxford Hospital Services Research Committee (AJ) and the UCL/UCLH NIHR Comprehensive Biomedical Research Centre (EJ).

\section{References:}

1. Rapoport JL, Giedd JN, Gogtay N. Neurodevelopmental model of schizophrenia: update 2012. Mol Psychiatry. 2012;17(12):1228-38. 
2. Eisenberg DP, Sarpal D, Kohn PD, Meyer-Lindenberg A, Wint D, Kolachana B, et al. Catechol-o-methyltransferase valine(158)methionine genotype and resting regional cerebral blood flow in medication-free patients with schizophrenia. Biol Psychiatry. 2010;67(3):287-90.

3. Gutierrez-Galve L, Wheeler-Kingshott CA, Altmann DR, Price G, Chu EM, Leeson VC, et al. Changes in the frontotemporal cortex and cognitive correlates in first-episode psychosis. Biol Psychiatry. 2010;68(1):51-60.

4. Nestor PG, Kubicki M, Niznikiewicz M, Gurrera RJ, McCarley RW, Shenton ME. Neuropsychological disturbance in schizophrenia: a diffusion tensor imaging study. Neuropsychology. 2008;22(2):246-54.

5. Kubicki M, Niznikiewicz M, Connor E, Nestor P, Bouix S, Dreusicke M, etal. Relationship Between White Matter Integrity, Attention, and Memory in Schizophrenia: A Diffusion Tensor Imaging Study. Brain Imaging Behav. 2009;3(2):191-201.

6. Karlsgodt KH, van Erp TG, Poldrack RA, Bearden CE, Nuechterlein KH, Cannon TD. Diffusion tensor imaging of the superior longitudinal fasciculus and working memory in recent-onset schizophrenia. Biol Psychiatry. 2008;63(5):512-8.

7. Quan M, Lee SH, Kubicki M, Kikinis Z, Rathi Y, Seidman LJ, et al. White matter tract abnormalities between rostral middle frontal gyrus, inferior frontal gyrus and striatum in first-episode schizophrenia. Schizophr Res. 2013;145(13):1-10.

8. Epstein KA, Cullen KR, Mueller BA, Robinson P, Lee S, Kumra S. White matter abnormalities and cognitive impairment in early-onset schizophrenia- 
spectrum disorders. J Am Acad Child Adolesc Psychiatry. 2014;53(3):362-72 e12.

9. Liu X, Lai Y, Wang X, Hao C, Chen L, Zhou Z, et al. Reduced white matter integrity and cognitive deficit in never-medicated chronic schizophrenia: A diffusion tensor study using TBSS. Behavioural brain research. 2013.

10. Sugranyes G, Kyriakopoulos M, Dima D, O'Muircheartaigh J, CorrigallR, Pendelbury G, et al. Multimodal analyses identify linked functional and white matter abnormalities within the working memory network in schizophrenia. Schizophr Res. 2012;138(2-3):136-42.

11. Simpson EH, Kellendonk C, Kandel E. A possible role for the striatum in the pathogenesis of the cognitive symptoms of schizophrenia. Neuron. 2010;65(5):585-96.

12. Behrens TE, Berg HJ, Jbabdi S, Rushworth MF, Woolrich MW.Probabilistic diffusion tractography with multiple fibre orientations: What can we gain? Neuroimage. 2007;34(1):144-55.

13. Behrens TE, Woolrich MW, Jenkinson M, Johansen-Berg H, Nunes RG, Clare S, et al. Characterization and propagation of uncertainty in diffusionweighted MR imaging. Magn Reson Med. 2003;50(5):1077-88.

14. Rajji TK, Ismail Z, Mulsant BH. Age at onset and cognition in schizophrenia: meta-analysis. Br J Psychiatry. 2009;195(4):286-93. 15. Lay B, Blanz B, Hartmann M, Schmidt MH. The psychosocial outcome of adolescent-onset schizophrenia: a 12-year followup. Schizophr Bull. 2000;26(4):801-16.

16. Kaufman J, Birmaher B, Brent D, Rao U, Flynn C, Moreci P, et al. Schedule for Affective Disorders and Schizophrenia for School-Age Children-Presentand 
Lifetime Version (K-SADS-PL): initial reliability and validity data. J Am Acad Child Adolesc Psychiatry. 1997;36(7):980-8.

17. Kay SR, Fiszbein A, Opler LA. The positive and negative syndrome scale (PANSS) for schizophrenia. Schizophr Bull. 1987;13(2):261-76.

18. Wechsler D. Wechsler Abbreviated Scale of Intelligence. San Antonio: The Psychological Corporation. 1999.

19. Cohen MJ. Children's Memory Scale. San Antonio: The Psychological Corporation. 1997.

20. Wechsler D. Wechsler Memory Scale-III San Antonio: The Psychological Corporation. 1997.

21. Meyers JE, Meyers KR. Rey Complex Figure Test and Recognition Trial. Odessa, FL: Psychological Assessment Resources, Inc. 1995.

22. Wechsler D. Wechsler Intelligence Scale for Children: Third Edition. New York: The Psychological Corporation. 1991.

23. Wechsler D. Wechsler Adult Intelligence Scale-Revised. Cleveland:The Psychological Corporation. 1981.

24. Delis D, Kaplan, E., Kramer, J. Delis-Kaplan Executive Function System. San Antonio: The Psychological Corporation. 2001.

25. Oldfield RC. The assessment and analysis of handedness: the Edinburgh inventory. Neuropsychologia. 1971;9(1):97-113.

26. Smith SM, Jenkinson M, Woolrich MW, Beckmann CF, Behrens TE, Johansen-Berg $\mathrm{H}$, et al. Advances in functional and structural MR image analysis and implementation as FSL. Neuroimage. 2004;23 Suppl 1:S208-19. 
27. Patenaude B. Bayesian Statistical Models of Shape and Appearance for Subcortical Brain Segmentation. Department of Neurology DPhil Thesis University of Oxford. 2007.

28. STATA. Stata/IC 12.1. http://www.stata.com;2012.

29. Smith SM, Nichols TE. Threshold-free cluster enhancement: addressing problems of smoothing, threshold dependence and localisation in cluster inference. Neuroimage. 2009;44(1):83-98.

30. Genovese CR, Lazar NA, Nichols T. Thresholding of statistical maps in functional neuroimaging using the false discovery rate. Neuroimage. 2002;15(4):870-8.

31. Lunn D, Spiegelhalter D, Thomas A, Best N. The BUGS project: Evolution, critique and future directions. Stat Med. 2009;28(25):3049-67.

32. Dickinson D, Ragland JD, Gold JM, Gur RC. General and specific cognitive deficits in schizophrenia: Goliath defeats David? Biol Psychiatry. 2008;64(9):823-7.

33. Draganski B, Kherif F, Kloppel S, Cook PA, Alexander DC, Parker GJ, etal. Evidence for segregated and integrative connectivity patterns in the human Basal Ganglia. J Neurosci. 2008;28(28):7143-52.

34. Laruelle M, Kegeles LS, Abi-Dargham A. Glutamate, dopamine, and schizophrenia: from pathophysiology to treatment. Annals of the NewYork Academy of Sciences. 2003;1003:138-58.

35. Ragland JD, Laird AR, Ranganath C, Blumenfeld RS, Gonzales SM, Glahn DC. Prefrontal activation deficits during episodic memory in schizophrenia. Am J Psychiatry. 2009;166(8):863-74. 
36. Eisenberg DP, Berman KF. Executive function, neural circuitry, and genetic mechanisms in schizophrenia. Neuropsychopharmacology. 2010;35(1):258-77.

37. Duncan J, Owen AM. Common regions of the human frontal lobe recruited by diverse cognitive demands. Trends Neurosci. 2000;23(10):475-83.

38. Duncan J. The multiple-demand (MD) system of the primate brain: mental programs for intelligent behaviour. Trends Cogn Sci. 2010;14(4):172-9.

39. Fry AF, Hale S. Relationships among processing speed, working memory, and fluid intelligence in children. Biol Psychol. 2000;54(1-3):1-34.

40. Schmithorst VJ, Wilke M, Dardzinski BJ, Holland SK. Cognitive functions correlate with white matter architecture in a normal pediatric population: a diffusion tensor MRI study. Hum Brain Mapp. 2005;26(2):139-47.

41. Mabbott DJ, Noseworthy M, Bouffet E, Laughlin S, Rockel C. White matter growth as a mechanism of cognitive development in children. Neuroimage. 2006;33(3):936-46.

42. Larsen B, Luna B. In vivo evidence of neurophysiological maturation of the human adolescent striatum. Dev Cogn Neurosci. 2014;12C:74-85.

43. Chakravarty MM, Rapoport JL, Giedd JN, Raznahan A, Shaw P, Collins DL, et al. Striatal shape abnormalities as novel neurodevelopmental endophenotypes in schizophrenia: A longitudinal study. Hum Brain Mapp. 2014.

44. Cohen MX, Schoene-Bake JC, Elger CE, Weber B. Connectivity-based segregation of the human striatum predicts personality characteristics. Nat Neurosci. 2009;12(1):32-4. 
45. Dickinson D, Ramsey ME, Gold JM. Overlooking the obvious: a metaanalytic comparison of digit symbol coding tasks and other cognitive measures in schizophrenia. Arch Gen Psychiatry. 2007;64(5):532-42.

46. Longman RS, Saklofske DH, Fung TS. WAIS-III percentile scores by education and sex for U.S. and Canadian populations. Assessment. 2007;14(4):426-32.

47. Torniainen M, Suvisaari J, Partonen T, Castaneda AE, Kuha A, Perala J, et al. Sex differences in cognition among persons with schizophrenia and healthy first-degree relatives. Psychiatry Res.

48. Tsai PC, McDowd J, Tang TC, Su CY. Processing speed mediates gender differences in memory in schizophrenia. The Clinical neuropsychologist. 2012;26(4):626-40.

49. Kyriakopoulos M, Frangou S. Recent diffusion tensor imaging findings in early stages of schizophrenia. Curr Opin Psychiatry. 2009;22(2):168-76.

50. Marenco S, Stein JL, Savostyanova AA, Sambataro F, Tan HY, Goldman AL, et al. Investigation of Anatomical Thalamo-Cortical Connectivity and fMRI Activation in Schizophrenia. Neuropsychopharmacology. 2011;37(2):499-507. 51. Alba-Ferrara LM, de Erausquin GA. What does anisotropy measure? Insights from increased and decreased anisotropy in selective fiber tracts in schizophrenia. Frontiers in integrative neuroscience. 2013;7:9.

52. Zhang D, Guo L, Hu X, Li K, Zhao Q, Liu T. Increased cortico-subcortical functional connectivity in schizophrenia. Brain Imaging Behav. 2012;6(1):27-35. 53. Sarpal DK, Robinson DG, Lencz T, Argyelan M, Ikuta T, Karlsgodt K, etal. Antipsychotic treatment and functional connectivity of the striatum in firstepisode schizophrenia. JAMA Psychiatry. 2015;72(1):5-13. 
54. Ozcelik-Eroglu E, Ertugrul A, Oguz KK, Has AC, Karahan S, Yazici MK. Effect of clozapine on white matter integrity in patients with schizophrenia: a diffusion tensor imaging study. Psychiatry Res. 2014;223(3):226-35. 
Table 1. Demographic and clinical details of adolescent-onset schizophrenia patients and healthy controls. Numbers are mean (standard deviation -SD). 


\begin{tabular}{|c|c|c|c|c|}
\hline & $\begin{array}{l}\text { Adolescent } \\
\text { Schizophrenia } \\
\text { patients }(n=37)\end{array}$ & $\begin{array}{l}\text { Controls } \\
(\mathrm{n}=24)\end{array}$ & Statistic & Significance \\
\hline Gender (Male/Female) & $23 / 14$ & $12 / 12$ & $\chi^{2} 0.88$ & $p=0.34$ \\
\hline Age $($ mean $\pm \mathrm{SD})$ & $16.3 \pm 1.1$ & $15.9 \pm 1.4$ & $\mathrm{t}_{59} 1.2$ & $p=0.22$ \\
\hline Handedness Left/Right & $6 / 31$ & $2 / 22$ & $\chi^{2} 0.79$ & $\mathrm{p}=0.37$ \\
\hline $\begin{array}{l}\text { Age at onset of symptoms } \\
\text { in years (mean } \pm \text { SD) }\end{array}$ & $14.5 \pm 1.5$ & - & & \\
\hline $\begin{array}{l}\text { Disease duration in years } \\
(\text { mean } \pm \mathrm{SD})\end{array}$ & $1.8 \pm 1.3$ & - & & \\
\hline $\begin{array}{l}\text { PANSS positive } \\
(\text { mean } \pm \mathrm{SD})\end{array}$ & $22.3 \pm 2.8$ & - & & \\
\hline $\begin{array}{l}\text { PANSS negative (mean } \pm \\
\text { SD) }\end{array}$ & $15.6 \pm 2.9$ & & & \\
\hline $\begin{array}{l}\text { Full Scale IQ (FSIQ) } \\
(\text { mean } \pm \text { SD) }\end{array}$ & $89.1 \pm 15.3$ & $108.8 \pm 13.9$ & $\mathrm{t}_{59} 5.1$ & $\mathrm{p}<0.001$ \\
\hline $\begin{array}{l}\text { Medication } \\
\text { chlorpromazine } \\
\text { equivalents }\end{array}$ & $345.6 \pm 230.1$ & & & \\
\hline
\end{tabular}




\begin{tabular}{|l|l|l|l|l|}
\hline Medication (number of & & & & \\
patients taking & 15 & & \\
Olanzapine & 9 & 5 & & \\
Risperidone & 1 & & \\
Quetiapine & 1 & & \\
Arpipirazole & 6 & & \\
Sulpiride & & & \\
Clozapine & & & \\
\hline
\end{tabular}


Table 2

\begin{tabular}{|c|c|c|c|c|}
\hline \multicolumn{5}{|c|}{ Volumetric data $\left(\mathrm{mm}^{3}\right)$ means and standard deviations ()} \\
\hline & & Control & Patients & $\mathrm{P}$ value \\
\hline & Cortical Grey Matter & $982559 \pm 17830$ & $898823 \pm 12805$ & 0.0001 \\
\hline & White Matter & $795998 \pm 20049$ & $736889 \pm 11807$ & 0.008 \\
\hline \multirow[t]{10}{*}{ Right } & Caudate & $2711 \pm 392$ & $2767 \pm 352$ & $\mathrm{n} / \mathrm{s}$ \\
\hline & Putamen & $3225 \pm 377$ & $3102 \pm 394$ & $\mathrm{n} / \mathrm{s}$ \\
\hline & Nucleus Accumbens & $240 \pm 80$ & $245 \pm 74$ & $\mathrm{n} / \mathrm{s}$ \\
\hline & Hippocampus & $2627 \pm 354$ & $2491 \pm 311$ & $\mathrm{n} / \mathrm{s}$ \\
\hline & Amygdala & $902 \pm 158$ & $865 \pm 242$ & $\mathrm{n} / \mathrm{s}$ \\
\hline & Motor Cortex & $72256 \pm 2202$ & $66840 \pm 977$ & 0.03 \\
\hline & Medial Oritofrontal Cortex & $8237 \pm 445$ & $7737 \pm 169$ & $\mathrm{n} / \mathrm{s}$ \\
\hline & Lateral Orbitofrontal Cortex & $15211 \pm 383$ & $14135 \pm 224$ & 0.03 \\
\hline & Dorsolateral Prefrontal Cortex & $66827 \pm 2232$ & $61655 \pm 1012$ & $\mathrm{n} / \mathrm{s}$ \\
\hline & Anterior Cingulate Cortex & $24402 \pm 606$ & $22644 \pm 451$ & $\mathrm{n} / \mathrm{s}$ \\
\hline \multirow[t]{10}{*}{ Left } & Caudate & $2654 \pm 482$ & $2687 \pm 432$ & $\mathrm{n} / \mathrm{s}$ \\
\hline & Putamen & $3200 \pm 331$ & $3190 \pm 368$ & $\mathrm{n} / \mathrm{s}$ \\
\hline & Nucleus Accumbens & $298 \pm 99$ & $295 \pm 70$ & $\mathrm{n} / \mathrm{s}$ \\
\hline & Hippocampus & $2527 \pm 391$ & $2455 \pm 336$ & $\mathrm{n} / \mathrm{s}$ \\
\hline & Amygdala & $937 \pm 155$ & $943 \pm 161$ & $\mathrm{n} / \mathrm{s}$ \\
\hline & Motor Cortex & $68137 \pm 1965$ & $62564 \pm 969$ & 0.02 \\
\hline & Medial Oritofrontal Cortex & $8279 \pm 511$ & $8109 \pm 180$ & $\mathrm{n} / \mathrm{s}$ \\
\hline & Lateral Orbitofrontal Cortex & $15609 \pm 351$ & $14368 \pm 231$ & 0.03 \\
\hline & Dorsolateral Prefrontal Cortex & $69357 \pm 2136$ & $63508 \pm 946$ & 0.027 \\
\hline & Anterior Cingulate Cortex & $21507 \pm 487$ & $19002 \pm 429$ & 0.008 \\
\hline
\end{tabular}




\begin{tabular}{lcccccc}
\hline \multicolumn{1}{c}{ Variable } & Coefficient & Mean & sd & $\mathbf{2 . 5 0 \%}$ & Median & $\mathbf{9 7 . 5 0 \%}$ \\
\cline { 2 - 5 } Intercept(C) & $\beta_{01}$ & 15.05 & 20.15 & -24.86 & 15.14 & 54.47 \\
Intercept(F) & $\beta_{02}$ & 55.62 & 9.243 & 36.89 & 55.77 & 73.37 \\
$\operatorname{sex}(\mathrm{C})$ & $\beta_{11}$ & 19.88 & 4.22 & 11.6 & 19.85 & 28.24 \\
$\operatorname{wm}(\mathrm{C})$ & $\beta_{31}$ & 7.615 & 2.395 & 2.938 & 7.601 & 12.37 \\
$\operatorname{canV1}(\mathrm{C})$ & $\beta_{41}$ & 27.75 & 11.52 & 4.749 & 27.87 & 50.16 \\
$\operatorname{canV1(F)}$ & $\beta_{42}$ & 46.08 & 10.69 & 24.52 & 46.23 & 66.62 \\
$\operatorname{canV2(C)}$ & $\beta_{51}$ & -38.01 & 11.67 & -60.7 & -38.1 & -14.7 \\
diagnosis & $\beta_{62}$ & 6.944 & 4.045 & -1.127 & 6.97 & 14.85 \\
Coding & $\alpha$ & 0.4719 & 0.1152 & 0.2489 & 0.47 & 0.705 \\
\hline
\end{tabular}

(C) effect on Coding.

(F) effect on FSIQ - full scale IQ.

Table 3. Bayesian Structural Model Coefficients 


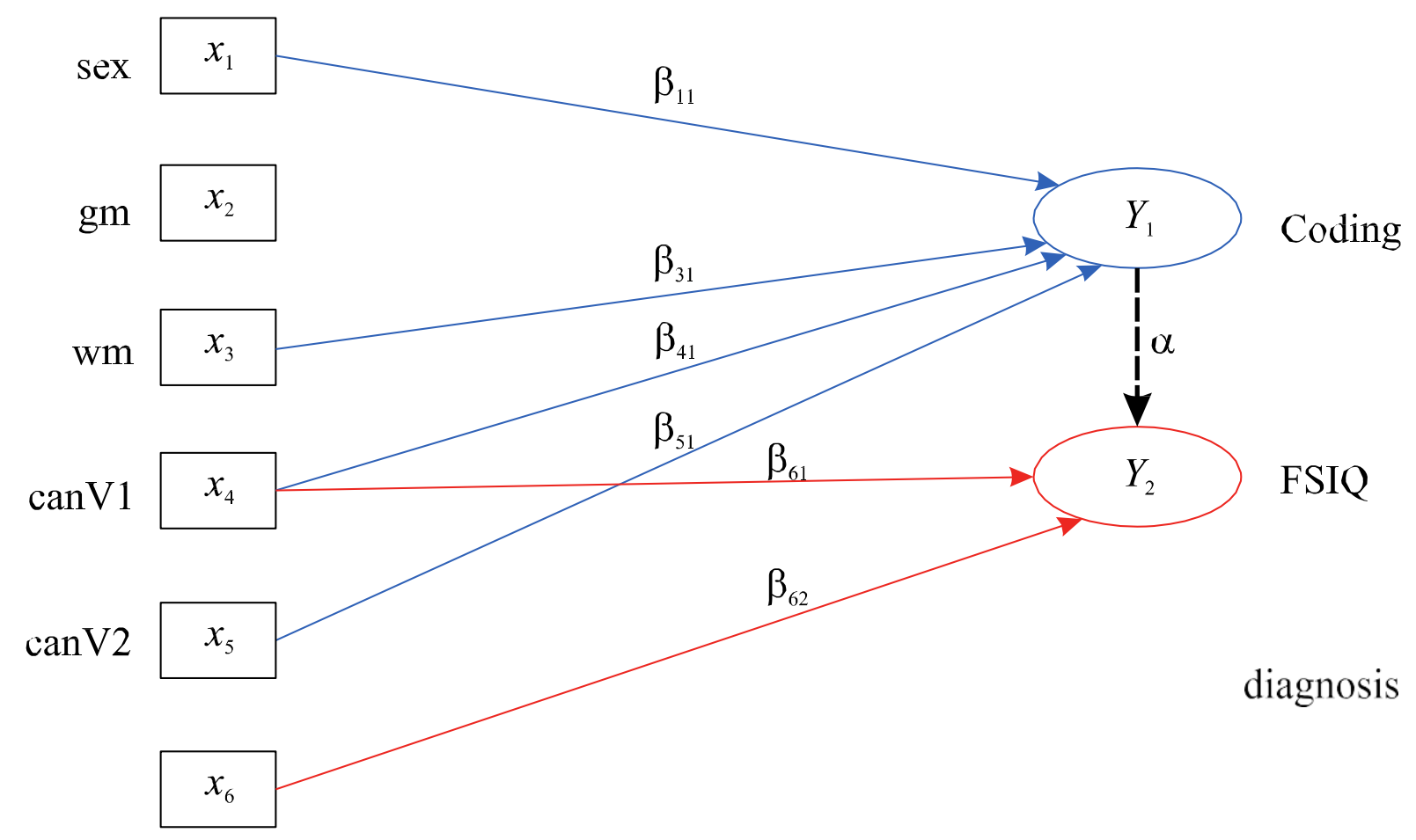

Figure 1 
Figure 2.

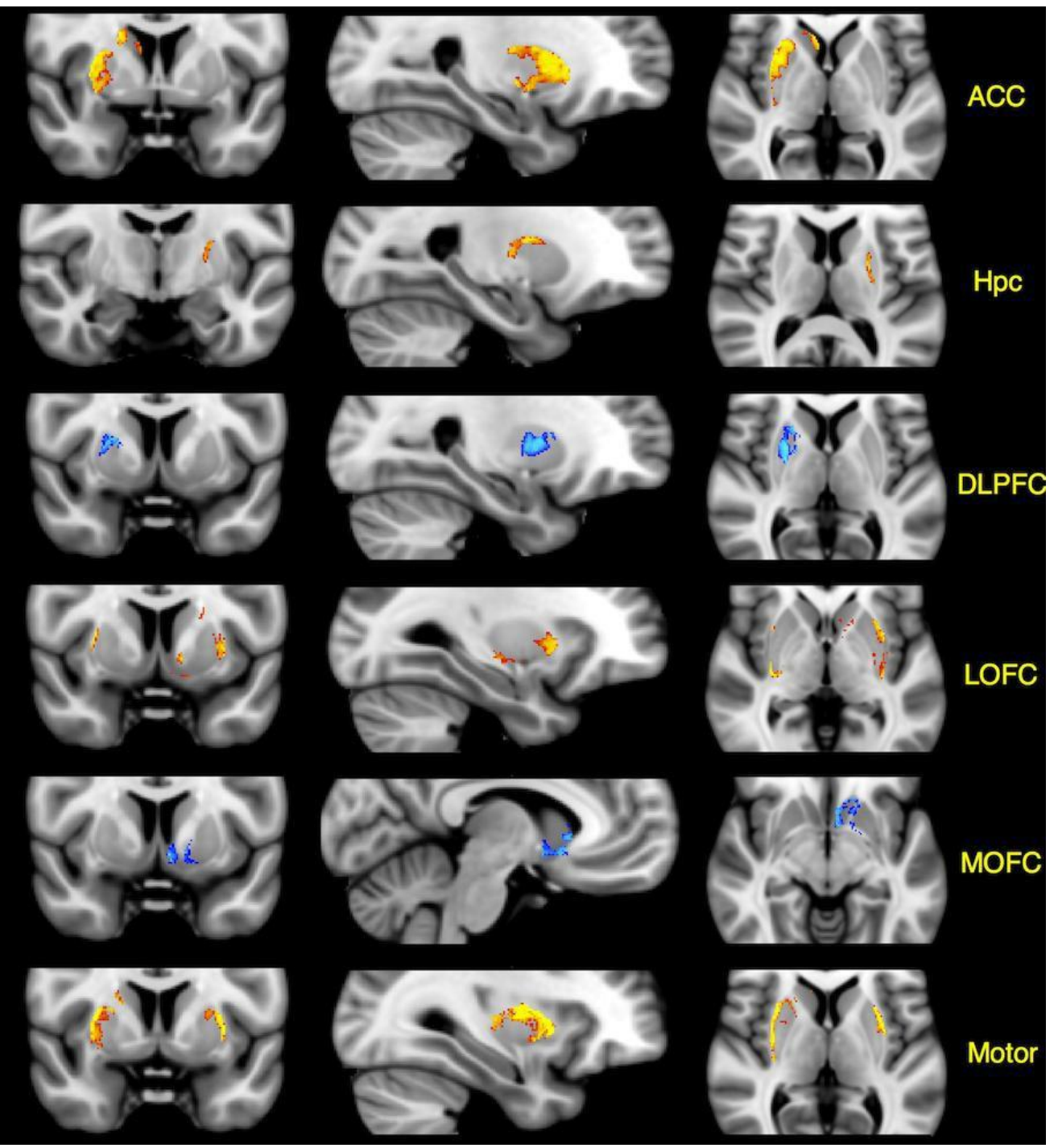


Figure 3.

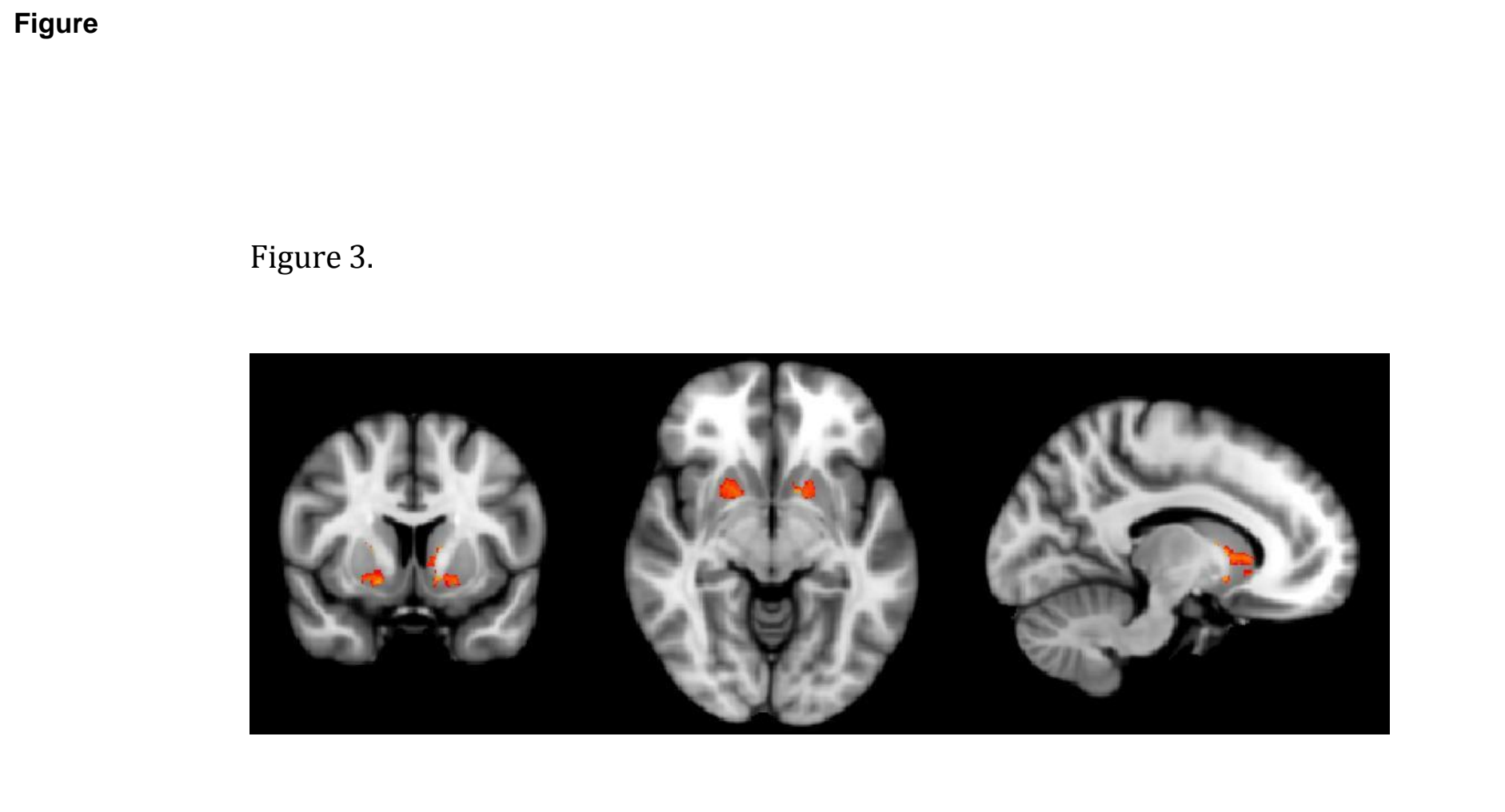

tras.

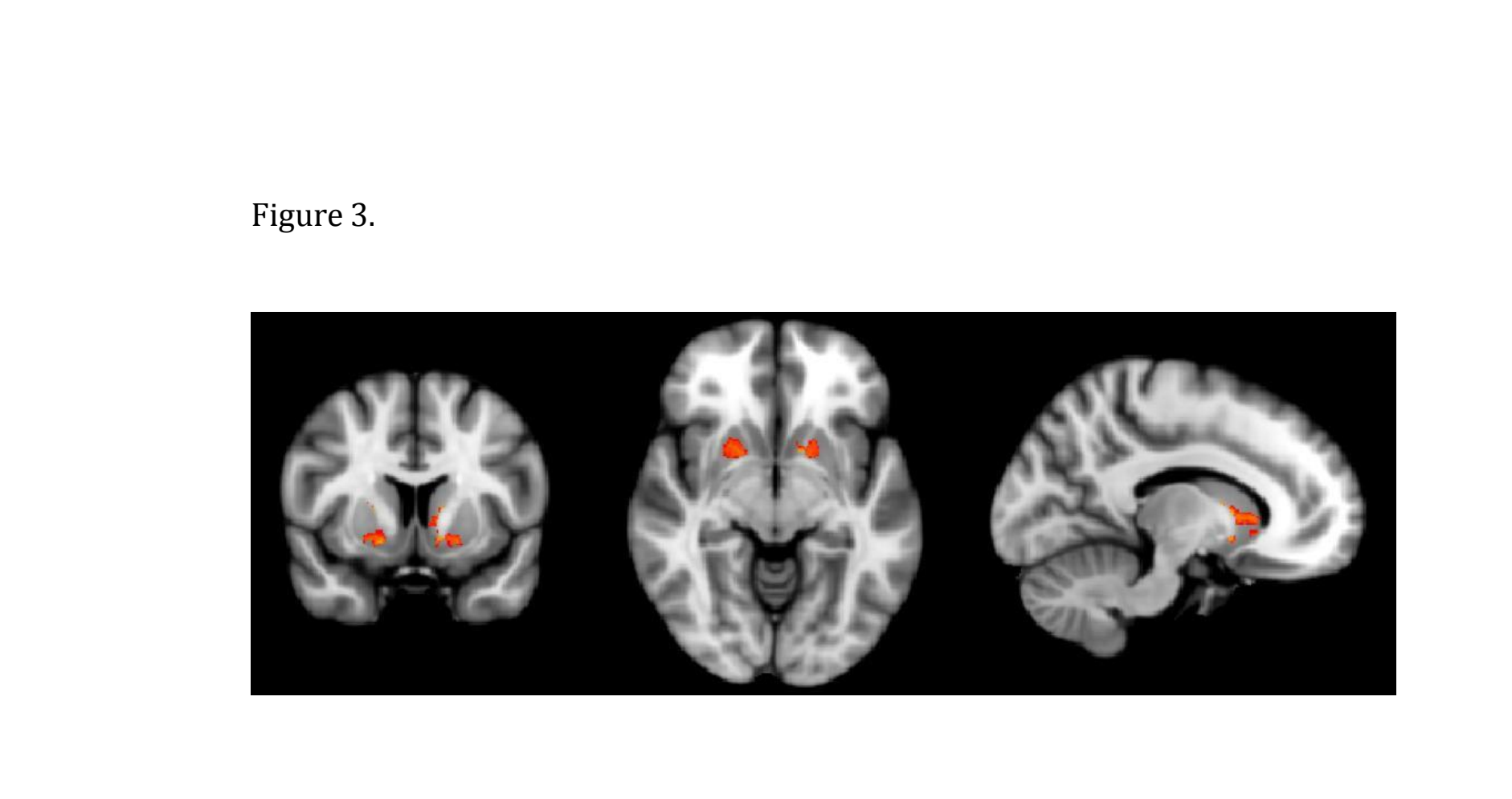

.
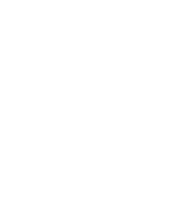
Figure 4

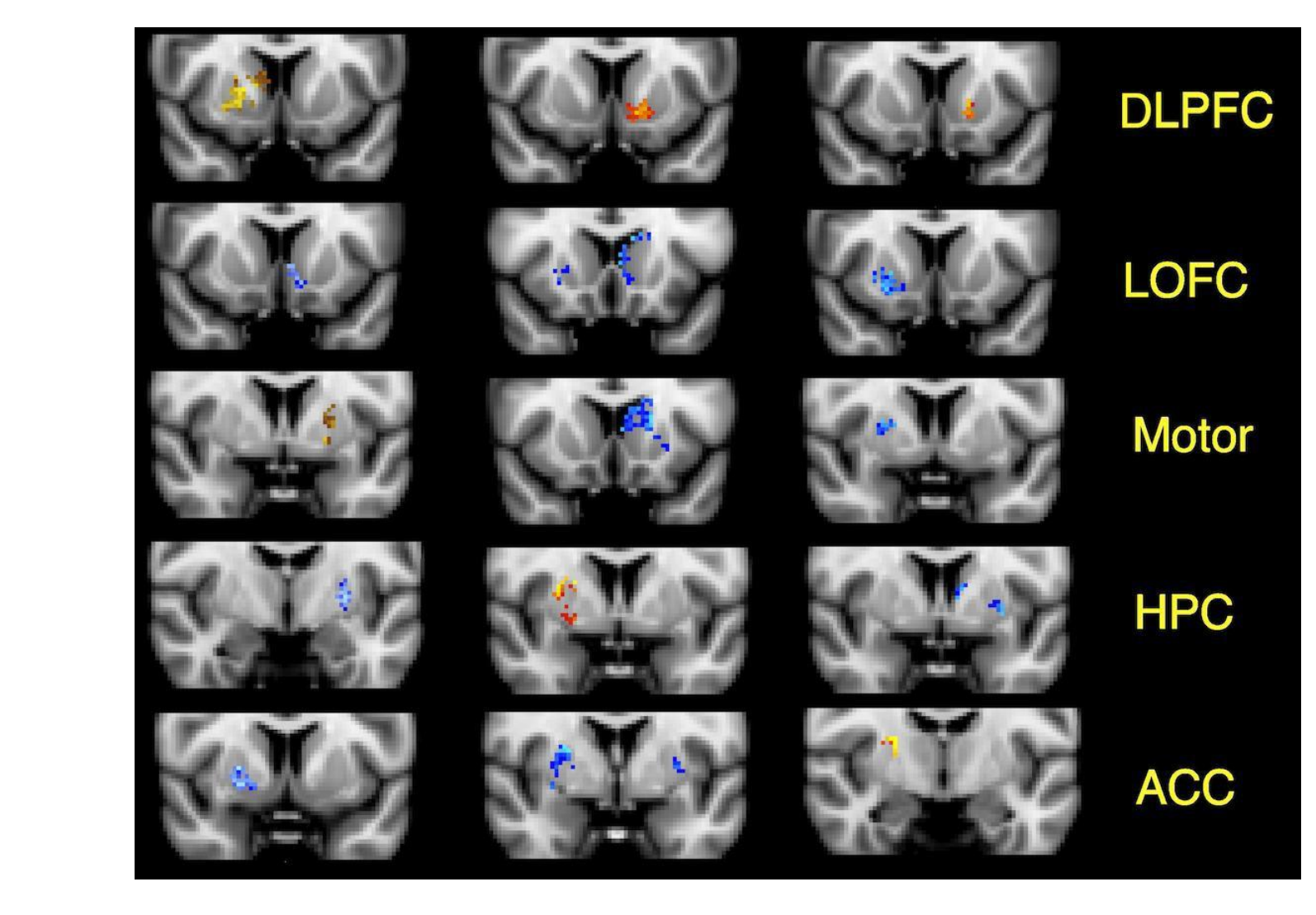

Figure 4

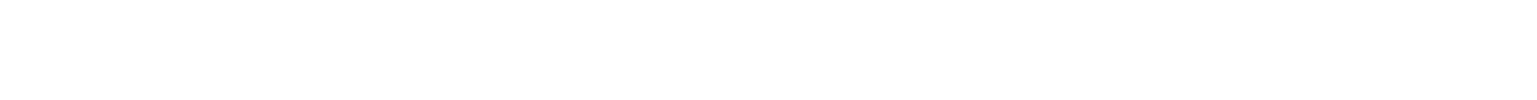

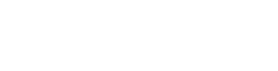




\section{Supplementary Table 1}

Volumetric data $\left(\mathrm{mm}^{3}\right)$ means and standard deviations()

$\begin{array}{llll} & \text { Control } & \text { Patients } & \text { P value } \\ \text { Cortical Grey Matter } & 982559 \pm 17830 & 898823 \pm 12805 & 0.0001 \\ \text { White Matter } & 795998 \pm 20049 & 736889 \pm 11807 & 0.008\end{array}$

\begin{tabular}{|c|c|c|c|c|}
\hline Right & Caudate & $2711 \pm 392$ & $2767 \pm 352$ & $\mathrm{n} / \mathrm{s}$ \\
\hline & Putamen & $3225 \pm 377$ & $3102 \pm 394$ & $\mathrm{n} / \mathrm{s}$ \\
\hline & Nucleus Accumbens & $240 \pm 80$ & $245 \pm 74$ & $\mathrm{n} / \mathrm{s}$ \\
\hline & Hippocampus & $2627 \pm 354$ & $2491 \pm 311$ & $\mathrm{n} / \mathrm{s}$ \\
\hline & Amygdala & $902 \pm 158$ & $865 \pm 242$ & $\mathrm{n} / \mathrm{s}$ \\
\hline & Motor Cortex & $72256 \pm 2202$ & $66840 \pm 977$ & 0.03 \\
\hline & Medial Oritofrontal Cortex & $8237 \pm 445$ & $7737 \pm 169$ & $\mathrm{n} / \mathrm{s}$ \\
\hline & Lateral Orbitofrontal Cortex & $15211 \pm 383$ & $14135 \pm 224$ & 0.03 \\
\hline & Dorsolateral Prefrontal Cortex & $66827 \pm 2232$ & $61655 \pm 1012$ & $\mathrm{n} / \mathrm{s}$ \\
\hline & Anterior Cingulate Cortex & $24402 \pm 606$ & $22644 \pm 451$ & $\mathrm{n} / \mathrm{s}$ \\
\hline Left & Caudate & $2654 \pm 482$ & $2687 \pm 432$ & $\mathrm{n} / \mathrm{s}$ \\
\hline & Putamen & $3200 \pm 331$ & $3190 \pm 368$ & $\mathrm{n} / \mathrm{s}$ \\
\hline & Nucleus Accumbens & $298 \pm 99$ & $295 \pm 70$ & $\mathrm{n} / \mathrm{s}$ \\
\hline & Hippocampus & $2527 \pm 391$ & $2455 \pm 336$ & $\mathrm{n} / \mathrm{s}$ \\
\hline & Amygdala & $937 \pm 155$ & $943 \pm 161$ & $\mathrm{n} / \mathrm{s}$ \\
\hline & Motor Cortex & $68137 \pm 1965$ & $62564 \pm 969$ & 0.02 \\
\hline & Medial Oritofrontal Cortex & $8279 \pm 511$ & $8109 \pm 180$ & $\mathrm{n} / \mathrm{s}$ \\
\hline
\end{tabular}


$\begin{array}{llll}\text { Lateral Orbitofrontal Cortex } \quad 15609 \pm 351 & 14368 \pm 231 & 0.03\end{array}$

$\begin{array}{llll}\text { Dorsolateral Prefrontal Cortex } & 69357 \pm 2136 & 63508 \pm 946 & 0.027\end{array}$

$\begin{array}{llll}\text { Anterior Cingulate Cortex } & 21507 \pm 487 & 19002 \pm 429 & 0.008\end{array}$ 


\begin{tabular}{lcc}
\hline & canV1 & canV2 \\
\hline ACC_L & 0.0514 & -0.0099 \\
AmyHpc_L & -0.0432 & -0.1356 \\
DLPFC_L & -0.0770 & 0.0404 \\
LOFC_L & 0.0277 & -0.1411 \\
MOFC_L & -0.0286 & 0.0614 \\
Motor_L & 0.0659 & -0.0333 \\
ACC_R & 0.0057 & 0.0733 \\
AmyHpc_R & 0.0286 & 0.0965 \\
DLPFC_R & -0.0575 & -0.0369 \\
LOFC_R & 0.1295 & 0.0701 \\
MOFC_R & -0.0399 & 0.0251 \\
Motor_R & 0.0827 & 0.0509 \\
\hline
\end{tabular}

Values standardised to zero mean and standard deviation 1

canV1 canV2 canonical variates ACC - anterior cingulate cortex; DLPFC dorsolateral prefrontal cortex; Hpc - Amygdalo-hippocampal complex; LOFC lateral orbitofrontal cortex; MOFC - medial orbitofrontal cortex; Motor - motor cortex.

Note The relative sizes of these coefficients give their relative contributions to canV1 and canV2. LOFC_R has a bigger contribution to make to canV1 than anything else with the Motor contribution coming next. In canV2, AmyHpc_L and LOFC_L are dominant.

Supplementary Table 2: Standardised loadings of fractional anisotropy (FA) from 12 tracts onto canonical variates canV1 and can V2. 